Gazi University
Journal of Science
http://dergipark.gov.tr/gujs

\title{
Parameter Estimation for Inverted Exponentiated Lomax Distribution with Right Censored Data
}

\author{
Amal HASSAN ${ }^{1}$ (D) , Rokaya MOHAMED ${ }^{1, *}$ \\ ${ }^{I}$ Department of Mathematical Statistics, Faculty of Graduate Studies for Statistical Research,Cairo University, Giza, Egypt
}

\section{Highlights}

- A new three-parameter model, called the inverted exponentiated Lomax distribution is proposed.

- Essential properties are studied.

- Based on Type I censoring, maximum likelihood estimators and asymptotic confidence intervals are provided.

- A simulation study is done to characterize the mean square errors of estimates for different sample sizes.

- A real data is used to illustrate the application and suitability of the proposed distribution.

\begin{tabular}{l} 
Article Info \\
\hline Received: $11 / 08 / 2018$ \\
Accepted: $19 / 02 / 2019$ \\
Keywords \\
\hline Exponentiated Lomax \\
distribution \\
Moments \\
Maximum likelihood \\
Type I Censoring
\end{tabular}

\section{INTRODUCTION}

The Lomax (L) distribution is a significant and widely used lifetime model. It has been employed in some areas, as; income, size of towns, queuing theory etc. It utilized for stochastic modeling of minimizing failure rate. The $\mathrm{L}$ distribution can be deduced as a special case from the compound gamma distribution [1]. The L distribution has been proposed as a substitutional to the exponential distribution for heavy-tailed data sets [2]. The $\mathrm{L}$ distribution has been applied in right censored data [3]. The record values of the L distribution have been proposed in $[4,5]$. Bayesian and non-Bayesian estimators of the sample size for L distribution, depending on Type-I censored (TIC) samples were discussed in [6]. The estimation of the L distribution under optimum step -stress accelerated life testing has been studied in [7]. The estimation of the L parameters depending on hybrid censoring samples has been considered in [8]. The estimation of the L distribution in accelerated life tests was discussed in [9]. Modified and extended versions of the L distribution are available such as; Marshall-Olkin extended-L distribution [10,11], exponentiated Lomax (EL) distribution [12], transmuted EL (TEL) distribution [13], extended Poisson-L distribution [14], exponential L distribution [15], Weibull L distribution [16], power L distribution [17]. Furthermore, EL geometric distribution, power L Poisson distribution, exponentiated Weibull L distribution and inverse power L distribution have been discussed in [18-21].

The cumulative distribution function (cdf) and probability density function (pdf) of a random variable $B$ has a L distribution, respectively, are 
$W(b ; \lambda, \alpha)=1-(1+\lambda b)^{-\alpha}, b, \lambda, \alpha>0$,

and

$$
w(b ; \lambda, \alpha)=\lambda \alpha(1+\lambda b)^{-(\alpha+1)}, b, \lambda, \alpha>0 .
$$

The EL has the following cdf and pdf (see [12]),

$$
W(b ; \lambda, \alpha, \theta)=\left\{1-(1+\lambda b)^{-\alpha}\right\}^{\theta}, b, \lambda, \alpha, \theta>0,
$$

and

$$
w(b ; \lambda, \alpha, \theta)=\lambda \alpha \theta(1+\lambda b)^{-(\alpha+1)}\left\{1-(1+\lambda b)^{-\alpha}\right\}^{\theta-1}, b, \lambda, \alpha, \theta>0 .
$$

In reliability studies, life-tests are performed to observe the life of the experimental units put on test. In such a life test, some surviving units are eliminated or lost owing to time, cost restrictions and instant needs of the units for other purposes. Censored samples are known as the incomplete data that obtained from a life-test. Censored samples provide only portion of the information about the failure time of the units under study. Consequently, this information should not be neglected or addressed as a failure. Good estimation parameters would not be possible to make and thus doing a proper analysis in the absence of such data. The conventional TIC and Type-II censoring (TIIC) are the two widespread censored samples. TIC data occur when every unit of a system are spotted up to the date of completion of the inspection. In TIC scheme, the test is terminated at the fixed time of examination. In TIIC scheme, the test is terminated at a pre-fixed number items have failed.

Lifetime distributions, in presence of censored sampling schemes, have been gained a great importance owing to their broad applications in disparate fields. So, our motivation here is to study the parameter estimation of the new three-parameter lifetime model, based on TIC samples. The new model is the inverse form of EL distribution; we call it the IEL. The remnant of the paper contains the following sections. The IEL distribution is provided in Section 2. Statistical properties are given in Section 3. Then, in Section 4, maximum likelihood (ML) and approximate confidence intervals (CIs) estimators under TIC samples are derived. Simulation studies are performed in Section 5. In addition, real data applications are performed in Section 6. The paper closed with a conclusion in Section 7.

\section{INVERTED EXPONENTIATED LOMAX DISTRIBUTION}

The importance of inverted distributions appears in applications related to many areas such as; econometrics, biological and engineering sciences, medical research and life testing. So, the main aim here is to introduce the IEL as the inverse form of the EL distribution.

The cdf of the IEL distribution, denoted by $\operatorname{IEL}(\lambda, \alpha, \theta)$, is derived, using the inverse transformation $\mathrm{Z}=1 / \mathrm{B}$, in (3) as follows

$$
F[z ; \lambda, \alpha, \theta]=1-\left\{1-[1+\lambda / z]^{-\alpha}\right\}^{\theta} ; \quad \lambda, \alpha, \theta, z>0
$$

The corresponding pdf is obtained as follows

$$
f[z ; \lambda, \alpha, \theta]=\alpha \theta\left[\lambda / z^{2}\right]\left\{1-[1+\lambda / z]^{-\alpha}\right\}^{\theta-1}[1+\lambda / z]^{-\alpha-1} ; \quad \lambda, \alpha, \theta, z>0 .
$$


The reliability function and hazard rate function (hrf) of the IEL distribution are given, respectively, as follows:

$$
R[z ; \lambda, \alpha, \theta]=\left\{1-[1+\lambda / z]^{-\alpha}\right\}^{\theta},
$$

and

$$
h[z ; \lambda, \alpha, \theta]=\frac{\alpha \theta \lambda[1+\lambda / z]^{-\alpha-1}}{z^{2}\left\{1-[1+\lambda / z]^{-\alpha}\right\}} .
$$

Further, the reversed-hrf and cumulative hrf of $Z$ are obtained as follows

$$
r[z ; \lambda, \alpha, \theta]=\frac{\alpha \theta \lambda\left\{1-[1+\lambda / z]^{-\alpha}\right\}^{\theta-1}[1+\lambda / z]^{-\alpha-1}}{z^{2}\left\{1-\left\{1-[1+\lambda / z]^{-\alpha}\right\}^{\theta}\right\}},
$$

and

$$
H[z ; \lambda, \alpha, \theta]=-\ln R[z ; \lambda, \alpha, \theta]=-\theta \ln \left\{1-[1+\lambda / z]^{-\alpha}\right\}
$$

Figures 1(a) and 1(b) display some potential shapes of the pdf and hrf of the IEL for different values of the parameters.

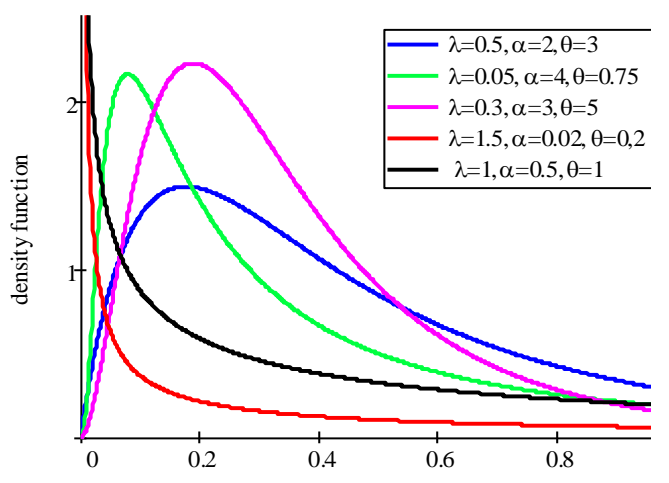

(a)

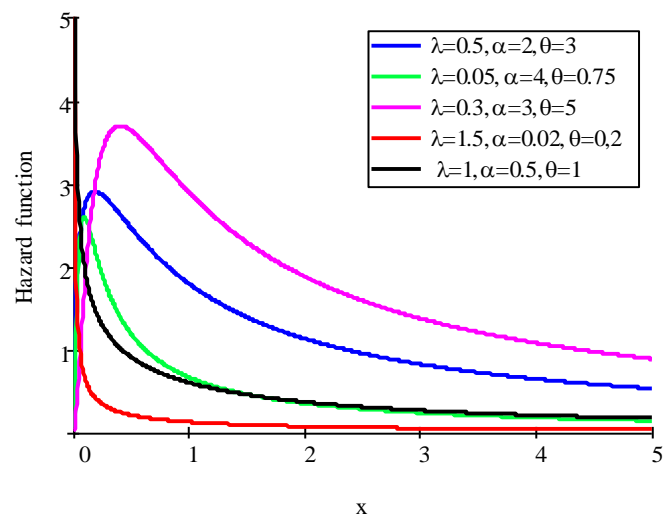

(b)

Figure 1. (a) the pdf plots and (b) the hrf plots of IEL for some selected values of parameters

It is clear from Figure 1(a) that the shapes of the IEL pdf are flexible for some selected parameter values. Also, as seen from Figure 1(b) that the behavior of the hrf are decreasing, reversed $\mathrm{J}$-shaped, increasing and up-side-down.

\section{STATISTICAL PROPERTIES}

Statistical properties of the IEL distribution including; moments, quantile measures, Rényi entropy, and distribution of order statistics (OS) are derived. 


\subsection{Moments}

The $k^{\text {th }}$ moment about zero for the IEL distribution, using pdf (6), is derived as follows

$\mu_{k}^{\prime}=\int_{0}^{\infty} z^{k} \alpha \theta\left[\lambda / z^{2}\right]\left\{1-[1+\lambda / z]^{-\alpha}\right\}^{\theta-1}[1+\lambda / z]^{-\alpha-1} d z$.

Let $y=\left(1+\frac{\lambda}{z}\right)^{-\alpha} \rightarrow d y=\alpha\left(1+\frac{\lambda}{z}\right)^{-\alpha-1} \frac{\lambda}{z^{2}} d z$.

Then Equation (7) convert to

$\mu_{k}^{\prime}=\theta \lambda^{k} \int_{0}^{1}\left[\frac{y^{\frac{1}{\alpha}}}{1-y^{\frac{1}{\alpha}}}\right]^{k}(1-y)^{\theta-1} d y$.

By using the geralized binomial series, then

$\mu_{k}^{\prime}=\theta \lambda^{k} \sum_{j=0}^{\infty} \frac{\Gamma(k+j)}{\Gamma(k) j !} \int_{0}^{1} y^{\frac{j+k}{\alpha}}(1-y)^{\theta-1} d y$,

which leads to

$\mu_{k}^{\prime}=\theta \lambda^{k} \sum_{j=0}^{\infty} \frac{\Gamma(k+j)}{\Gamma(k) j !} \frac{\Gamma((j+k / \alpha)+1) \Gamma(\theta)}{\Gamma((j+k / \alpha)+\theta+1)}, k=1,2, \ldots$

The $k^{\text {th }}$ central moment $\left(\mu_{k}\right)$ of $Z$ is given by

$\mu_{k}=E\left\{Z-\mu_{1}^{\prime}\right\}^{k}=\sum_{i=0}^{k}[-1]^{i}\left(\begin{array}{c}k \\ i\end{array}\right)\left(\mu_{1}^{\prime}\right)^{i} \mu_{k-i}^{\prime}$.

To check how the mean and variance change for different parameters values, numerical results are provided via Mathcad (14). Table 1 gives the mean and variance of the IEL distribution for diverse parameter values. From Table 1, it can be detected that both values of the mean and variance of the IEL decrease as the values of $\theta$ increase and increase as the values of $\lambda$ and $\alpha$ increase. 
Table 1. Mean and variance of IEL distribution for diverse values of $\alpha, \lambda$ and $\theta$

\begin{tabular}{|c|c|c|c|c|c|c|c|c|c|}
\hline & \multirow[b]{2}{*}{$\lambda$} & \multicolumn{2}{|l|}{$\theta=3$} & \multicolumn{2}{|l|}{$\theta=4$} & \multicolumn{2}{|c|}{$\theta=4.5$} & \multicolumn{2}{|l|}{$\theta=5$} \\
\hline & & $\mu_{1}^{\prime}$ & $\sigma_{1}^{2}$ & $\mu_{1}^{\prime}$ & $\sigma_{1}^{2}$ & $\mu_{1}^{\prime}$ & $\sigma_{1}^{2}$ & $\mu_{1}^{\prime}$ & $\sigma_{1}^{2}$ \\
\hline \multirow{3}{*}{0.5} & 1 & 0.159 & 0.157 & 0.09 & 0.039 & 0.072 & 0.023 & 0.059 & 0.015 \\
\hline & 2 & 0.318 & 0.628 & 0.181 & 0.154 & 0.144 & 0.093 & 0.118 & 0.06 \\
\hline & 3 & 0.477 & 1.413 & 0.271 & 0.347 & 0.217 & 0.209 & 0.178 & 0.136 \\
\hline \multirow{3}{*}{1.5} & 1 & 0.891 & 1.771 & 0.628 & 0.554 & 0.552 & 0.376 & 0.494 & 0.273 \\
\hline & 2 & 1.782 & 7.084 & 1.256 & 2.214 & 1.103 & 1.503 & 0.987 & 1.093 \\
\hline & 3 & 2.673 & 15.94 & 1.885 & 4.982 & 1.655 & 3.381 & 1.481 & 2.458 \\
\hline \multirow{3}{*}{3.5} & 1 & 2.564 & 10.011 & 1.928 & 3.265 & \begin{tabular}{|l}
1.739 \\
\end{tabular} & 2.263 & 1.595 & 1.679 \\
\hline & 2 & 5.128 & 40.042 & 3.855 & 13.058 & 3.479 & 9.051 & 3.19 & 6.717 \\
\hline & 3 & 7.691 & 90.095 & 5.783 & 29.381 & 5.218 & 20.365 & 4.785 & 15.114 \\
\hline
\end{tabular}

Furthermore, we can get the moment generating function from moments in such a way, where, it is easy to show that

$M_{Z}(t)=\sum_{k=0}^{\infty} \frac{t^{k} \mu_{k}^{\prime}}{\Gamma(k+1)}=\sum_{j, k=0}^{\infty} \frac{t^{k} \Gamma(k+j)}{\Gamma(k+1) \Gamma(k) j !} \theta \lambda^{k} \frac{\Gamma((j+k / \alpha)+1) \Gamma(\theta)}{\Gamma((j+k / \alpha)+\theta+1)}, k=1,2, \ldots$

One can obtain the moments about zero, $\mu_{1}^{\prime}, \mu_{2}^{\prime}, \ldots$ from the previous equation, where, $\mu_{1}^{\prime}=M_{Z}^{\prime}(0), \mu_{2}^{\prime}=M_{Z}^{\prime \prime}(0), \ldots$

\subsection{Quantile Function}

We can determine the quantile function of $Z \sim \operatorname{IEL}(\lambda, \alpha, \theta)$ with $\operatorname{pdf}(6)$, where; $Q(u)=F^{-1}(u)$ as:

$Q(u)=\lambda\left(\left[1-(1-u)^{\frac{1}{\theta}}\right]^{\frac{-1}{\alpha}}-1\right)^{-1}$,

where, $u$, has the uniform random variable in the interval $(0,1)$. Individually, the first quartile, second and third quartile are obtained by substituting $u=0.25,0.5$ and $u=0.75$ in (9). The Bowley skewness ( $B S$ ); (see [22]), based on quantiles, is given by

$$
B S=\{Q(0.75)-2 Q(0.5)+Q(0.25)\} /\{Q(0.75)-Q(0.25)\}
$$

Further, the Moors kurtosis ( $M K$ ); (see [23]) is defined as

$$
M K=\{Q(0.875)-Q(0.625)+Q(0.375)-Q(0.125)\} /\{Q(0.75)-Q(0.25)\}
$$

where $Q($.$) denotes the quantile function. The graphs of B S$ and $M K$ are given below for different values of the parameters. Figures 2 and 3 display plots of $B S$ and $M K$ for selected values of $\theta$ as function of $\alpha$ and for selected values of $\alpha$ as function of $\theta$. These plots demonstrate that the $B S$ reduces for increasing 
value of $\theta$ for fixed $\alpha$ and when the value of $\alpha$ increases for fixed $\theta$. From these figures, we reveal that the $M K$ curves have considerable flexibility.
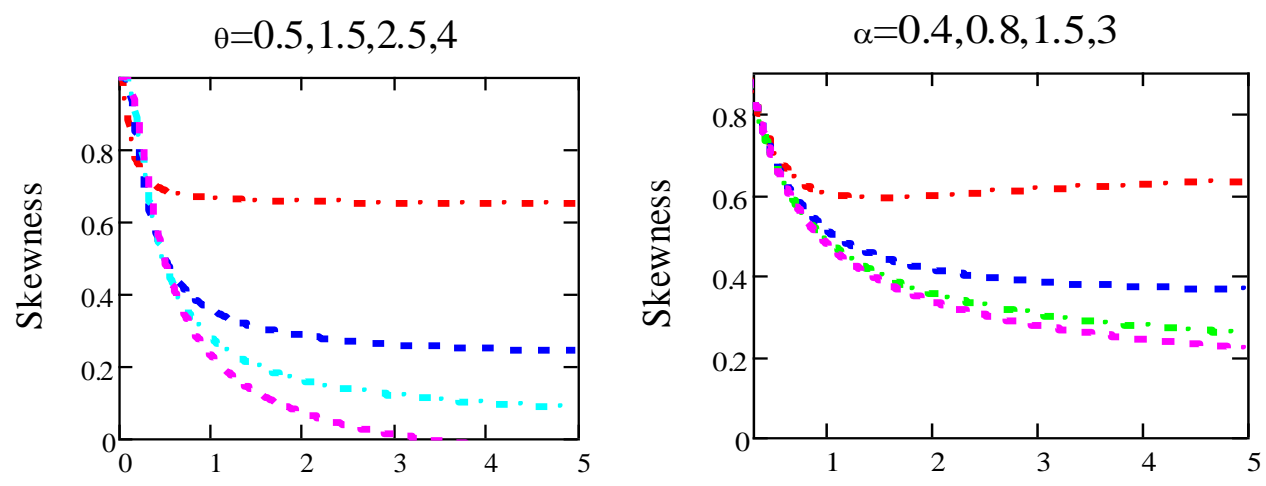

$\theta$

Figure 2. Skewness of the IEL with different values of $\alpha$ and $\theta$
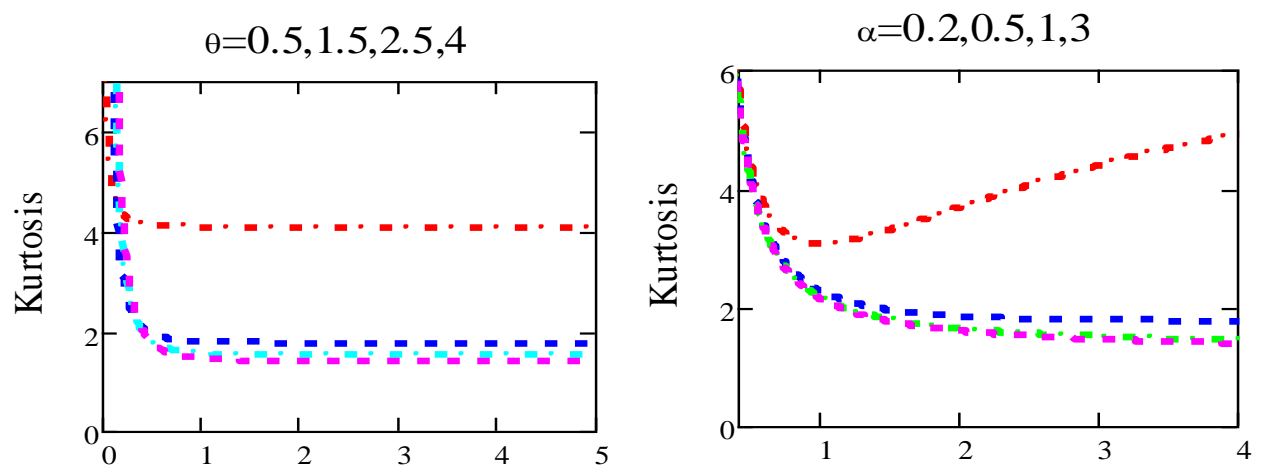

$\theta$

Figure 3. Kurtosis of the IEL with different values of $\alpha$ and $\theta$

\subsection{Rényi Entropy}

Entropy has been utilized in disparate directions, for instance; science and engineering. Furthermore, it is a measure of variation of the uncertainty. The Rényi entropy of a random variable $Z$, for $\delta>0$, and $\delta \neq 1$, is formulated as

$$
I_{R}[z]=[1-\delta]^{-1} \log \left(\int_{0}^{\infty}[f(z ; \lambda, \alpha, \theta)]^{\delta} d z\right)
$$

The Rényi entropy of IEL distribution is obtained by inserting pdf (6) in (10) as follows

$$
I_{R}[z]=[1-\delta]^{-1} \log \left(\int_{0}^{\infty}\left(\alpha \theta\left[\lambda / z^{2}\right]\right)^{\delta}\left\{1-[1+\lambda / z]^{-\alpha}\right\}^{\delta(\theta-1)}[1+\lambda / z]^{-\delta(\alpha+1)} d z\right)
$$

Using binomial expansion, we obtain

$$
I_{R}[z]=[1-\delta]^{-1} \log \left(\sum_{j=0}^{\infty}[-1]^{j}\left(\begin{array}{c}
\delta(\theta-1) \\
j
\end{array}\right)(\alpha \theta \lambda)^{\delta} \int_{0}^{\infty} z^{-2 \delta}[1+\lambda / z]^{-\alpha j-\delta(\alpha+1)} d z\right)
$$

After simplification, the formula becomes 


$$
I_{R}[z]=[1-\delta]^{-1} \log \left(\sum_{j=0}^{\infty}[-1]^{j}\left(\begin{array}{c}
\delta(\theta-1) \\
j
\end{array}\right)(\alpha \theta)^{\delta} \lambda^{1-2 \delta} \frac{\Gamma(2 \delta-1) \Gamma(\alpha j+\delta(\alpha+1)-2 \delta+1)}{\Gamma(\alpha j+\delta(\alpha+1))}\right) .
$$

\subsection{Distribution of Order Statistics}

The pdf of the qth OS of the IEL distribution is determined. Let $Z_{(1)}<Z_{(2)}, \ldots<Z_{(n)}$ be the OS for a random sample $Z_{1}, Z_{2}, \ldots, Z_{n}$ of size $n$ from the IEL distribution. It is recognized that, the pdf of the $q^{\text {th }}$ OS (see [24]) is defined by

$$
f_{(q)}[z]=\frac{1}{B(q, n-q+1)}\{F[z]\}^{q-1}\{1-F[z]\}^{n-q} f[z] .
$$

Utilizing the binomial series expansion for $\{1-F[z]\}^{n-q}$, then, $f_{(q)}[z]$ becomes

$$
f_{(q)}[z]=\frac{1}{B(q, n-q+1)} \sum_{m=0}^{n-q}[-1]^{m}\left(\begin{array}{c}
n-q \\
m
\end{array}\right)\{F[z]\}^{q+m-1} f[z] .
$$

Inserting cdf (5) and pdf (6) in (11), we obtain

$$
\begin{aligned}
f_{(q)}[z ; \lambda, \alpha, \theta] & =\frac{1}{B(q, n-q+1)} \sum_{m=0}^{n-q} \sum_{i=0}^{q+m-1}[-1]^{m+i}\left(\begin{array}{c}
q+m-1 \\
i
\end{array}\right)\left(\begin{array}{c}
n-q \\
m
\end{array}\right) \alpha \theta\left[\lambda / z^{2}\right] \times \\
\left\{1-[1+\lambda / z]^{-\alpha}\right\}^{\theta+\theta i-1}[1+\lambda / z]^{-\alpha-1} &
\end{aligned}
$$

As specifically, the pdf of the smallest OS; $Z_{(1)}$, can be obtained as:

$$
f_{(1)}[z ; \lambda, \alpha, \theta]=n \sum_{m=0}^{n-1} \sum_{i=0}^{m}[-1]^{m+i}\left(\begin{array}{c}
m \\
i
\end{array}\right)\left(\begin{array}{c}
n-1 \\
m
\end{array}\right) \alpha \theta\left[\lambda / z^{2}\right]\left\{1-[1+\lambda / z]^{-\alpha}\right\}^{\theta+\theta i-1}[1+\lambda / z]^{-\alpha-1} .
$$

As well, the pdf of the largest OS; $Z_{(n)}$, can be obtained as:

$$
f_{(n)}[z ; \lambda, \alpha, \theta]=n \sum_{i=0}^{n+m-1}[-1]^{m+i}\left(\begin{array}{c}
n+m-1 \\
i
\end{array}\right) \alpha \theta\left[\lambda / z^{2}\right]\left\{1-[1+\lambda / z]^{-\alpha}\right\}^{\theta+\theta i-1}[1+\lambda / z]^{-\alpha-1} .
$$

\section{PARAMETER ESTIMATION}

The point and approximate CI estimators of the IEL population parameters, under TIC scheme, are obtained using ML technique.

Let $Z_{(1)}<Z_{(2)}<\ldots<Z_{(r)}$ be a TIC sample of size $r$ whose life time's follow the IEL distribution (6) are placed on a life test and the test is stopped at specified time $T$ before all $n$ items have failed. The loglikelihood function, based on TIC, is 


$$
\begin{aligned}
\ln l=\ln & {[n ! /(n-r) ! r !]+r \ln \alpha+r \ln \theta+r \ln \lambda-2 \sum_{i=1}^{r} \ln z_{(i)}-[\alpha+1] \sum_{i=1}^{r} \ln S_{(i)}+[\theta-1] \sum_{i=1}^{r} \ln \left[1-S_{(i)}^{-\alpha}\right]+\theta[n-r] \times } \\
& \ln \left[1-(1+\lambda / T)^{-\alpha}\right] .
\end{aligned}
$$

where, $S_{(i)}=\left(1+\lambda / z_{(i)}\right)$, so for simplicity, we write $S_{i}$ instead of $S_{(i)}$. The partial derivatives with respect to the parameters are obtained as:

$$
\begin{gathered}
\partial \ln l / \partial \alpha=[r / \alpha]-\sum_{i=1}^{r} \ln S_{i}+[\theta-1] \sum_{i=1}^{r}\left(S_{i}\right)^{-\alpha} \ln \left(S_{i}\right)\left[1-\left(S_{i}\right)^{-\alpha}\right]^{-1}+[n-r] \theta(1+\lambda / T)^{-\alpha} \ln (1+\lambda / T) \times \\
{\left[1-(1+\lambda / T)^{-\alpha}\right]^{-1},} \\
\partial \ln l / \partial \lambda=[r / \lambda]-[\alpha+1] \sum_{i=1}^{r}\left(S_{i} z_{i}\right)^{-1}+[\theta-1] \alpha \sum_{i=1}^{r}\left(S_{i}\right)^{-\alpha-1}\left[1-\left(S_{i}\right)^{-\alpha}\right]^{-1} z_{i}^{-1}+[n-r] \theta \alpha(1+\lambda / T)^{-\alpha-1} \times \\
T^{-1}\left[1-(1+\lambda / T)^{-\alpha}\right]^{-1},
\end{gathered}
$$

and

$$
\partial \ln l / \partial \theta=[r / \theta]+\sum_{i=1}^{r} \ln \left[1-\left(S_{i}\right)^{-\alpha}\right]+[n-r] \ln \left[1-(1+\lambda / T)^{-\alpha}\right] .
$$

Then the ML estimators of the population parameters are the solution of non-linear Equations (13) - (15) after setting them equal zeros. These equations are very difficult to obtain, so iterative procedures are used. Further, in case of interval estimation, the $3 \times 3$ observed information matrix $I(\Phi)=\left\{I_{u v}\right\}$ for $(u, v)=(\alpha, \lambda, \theta)$ is considered. It is known that, under the regularity condition, the asymptotic properties of the ML method ensure that: $\sqrt{n}(\hat{\Phi}-\Phi) \stackrel{d}{\longrightarrow} N_{3}\left(0, I^{-1}(\Phi)\right)$ as $n \rightarrow \infty$ where $\stackrel{d}{\longrightarrow}$ means the convergence in distribution, with mean $0=(0,0,0)^{T}$ and $3 \times 3$ variance-covariance matrix $I^{-1}(\Phi)$ then, the $100(1-v) \%$ CIs for $\alpha, \lambda$, and $\theta$ are given, respectively, as follows

$$
\hat{\alpha} \pm Z_{\frac{v}{2}} \sqrt{\sigma^{2}(\hat{\alpha})}, \hat{\lambda} \pm Z_{\frac{v}{2}} \sqrt{\sigma^{2}(\hat{\lambda})} \text { and } \hat{\theta} \pm Z_{\frac{v}{2}} \sqrt{\sigma^{2}(\hat{\theta})}
$$

where $Z_{\frac{v}{2}}$ is the $[100(1-v / 2)]$ th standard normal percentile and $\sigma^{2}$ 's denote the diagonal elements of $I^{-1}(\Phi)$ corresponding to the model parameters.

\section{SIMULATION STUDY}

In this section, a numerical study is presented to examine the behavior of the estimators for different parameter values. The behavior of the estimates of unknown parameters is measured by their mean square errors (MSEs), relative biases (RBs), standard errors (SEs), lower confidence bound (LCB), upper confidence bound (UCB), and length of $95 \%$ CIs. The numerical procedures are formed as follows: 
Step (1): 1000 random sample of sizes 50, 100, 150, 200, 250 and 300 are selected, these random samples are generated from the IEL distribution.

Step (2): Values of the unknown parameters $(\alpha, \lambda, \theta)$ are selected as $\operatorname{Set} 1=(\alpha=0.5, \lambda=0.8, \theta=2)$, $\operatorname{Set} 2=(\alpha=0.3, \lambda=2, \theta=1.5), \operatorname{Set} 3=(\alpha=1.5, \lambda=0.5, \theta=0.4)$ and $\operatorname{Set} 4=(\alpha=2, \lambda=1.5, \theta=0.8)$. The termination time is selected as $T=0.3$.

Step (3): The MSEs, RBs, SEs for all samples sizes and for all selected sets of parameters are computed. Furthermore, the LCB, UCB and length with confidence level 0.95 for all samples sizes and for all selected sets of parameters are calculated.

Numerical outcomes are reported in Tables 2 to 5. Based on these tables, we can detect the following about the performance of the estimated parameters:

1. For all sets of parameters, SEs of all parameters decrease as the sample sizes increase (see Tables 2, 3 and Figure 5).

2. The MSEs and RBs of $\alpha, \lambda$ and $\theta$ decrease as the sample sizes increase for different selected sets of parameters (see Tables 2, 3 and Figure 4).

3. The MSEs and SEs of $\theta$ are smaller than the corresponding MSEs and SEs for the other estimates of $\alpha$ and $\lambda$ in almost all of the cases (see Table 2).

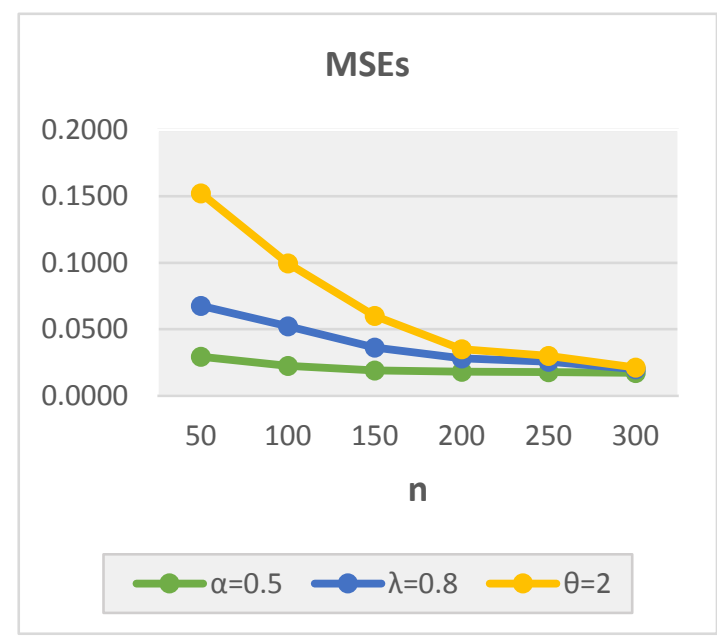

Figure 4. MSEs for Set1

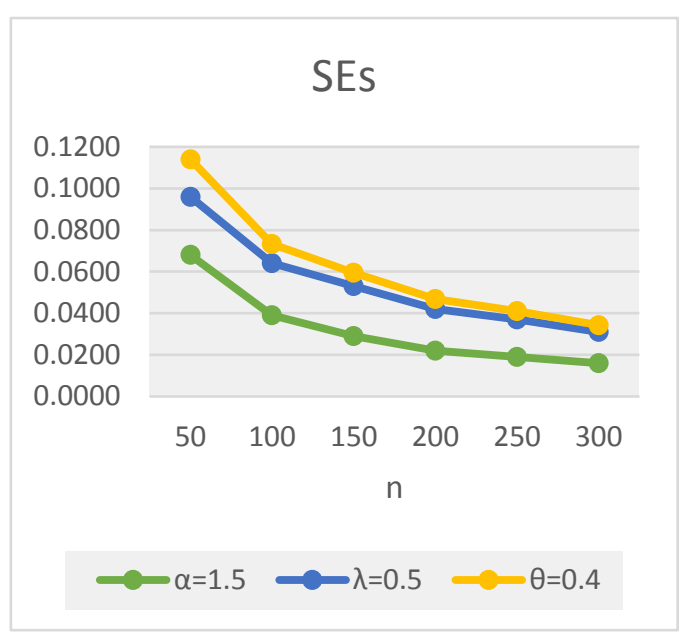

Figure 5. SEs for Set3

4. As it seems from Figure 6, the SEs of $\alpha$ for all sets of parameters have the smallest values for the same sample size. Also, it is clear that Set 1 has the smallest SEs corresponding to the other sets of parameters.

5. For all sets, it is clear that the length of CIs for the model parameters decreases as sample size increases (see Tables 4, 5 and Figure 7).

6. As it seems from Figure 7, the length of $\alpha, \lambda$ and $\theta$ decreases as the sample sizes increase for different selected sets of parameters (see also, Tables 4 and 5). 


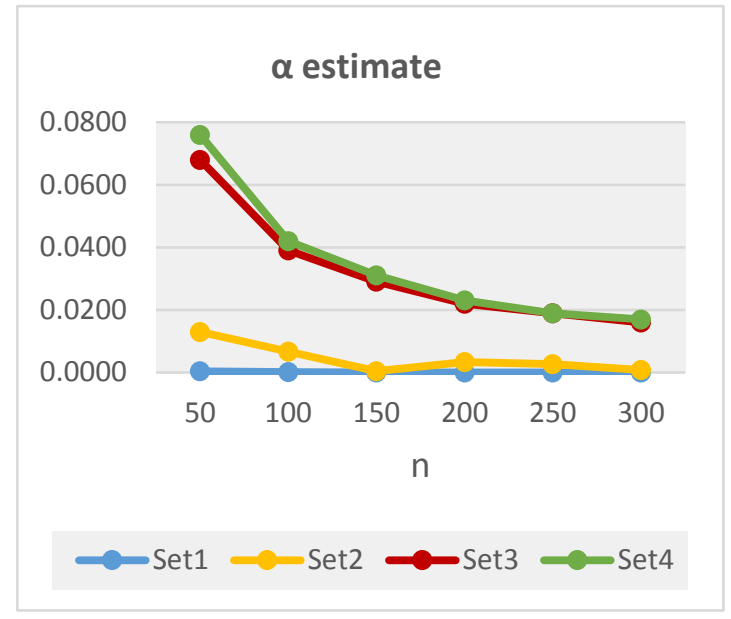

Figure 6. SEs of $\alpha$ for all set of parameters

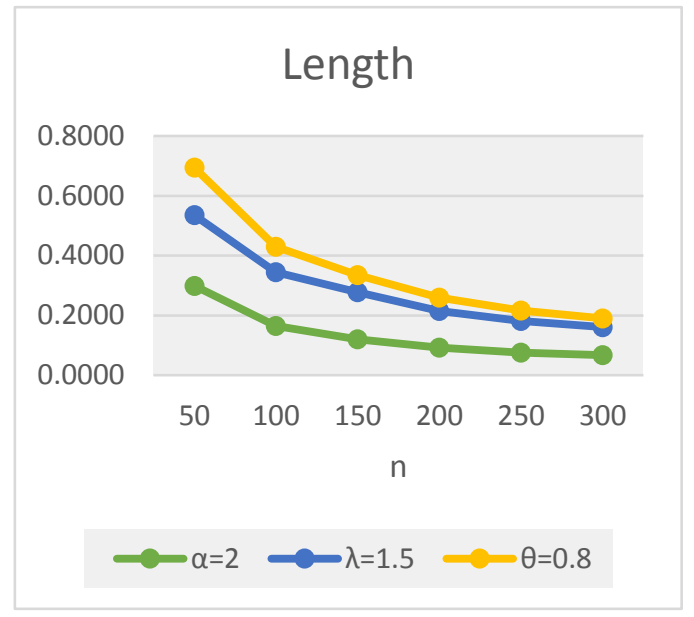

Figure 7. Lengths for Set4

7. As it seems from Figures 8 and 9, the MSEs of $\lambda$ and $\theta$ for all sets have the smallest values for the same sample size. Also, it is clear that the Sets 1 and 2 have the smallest MSEs corresponding to other sets of parameters.
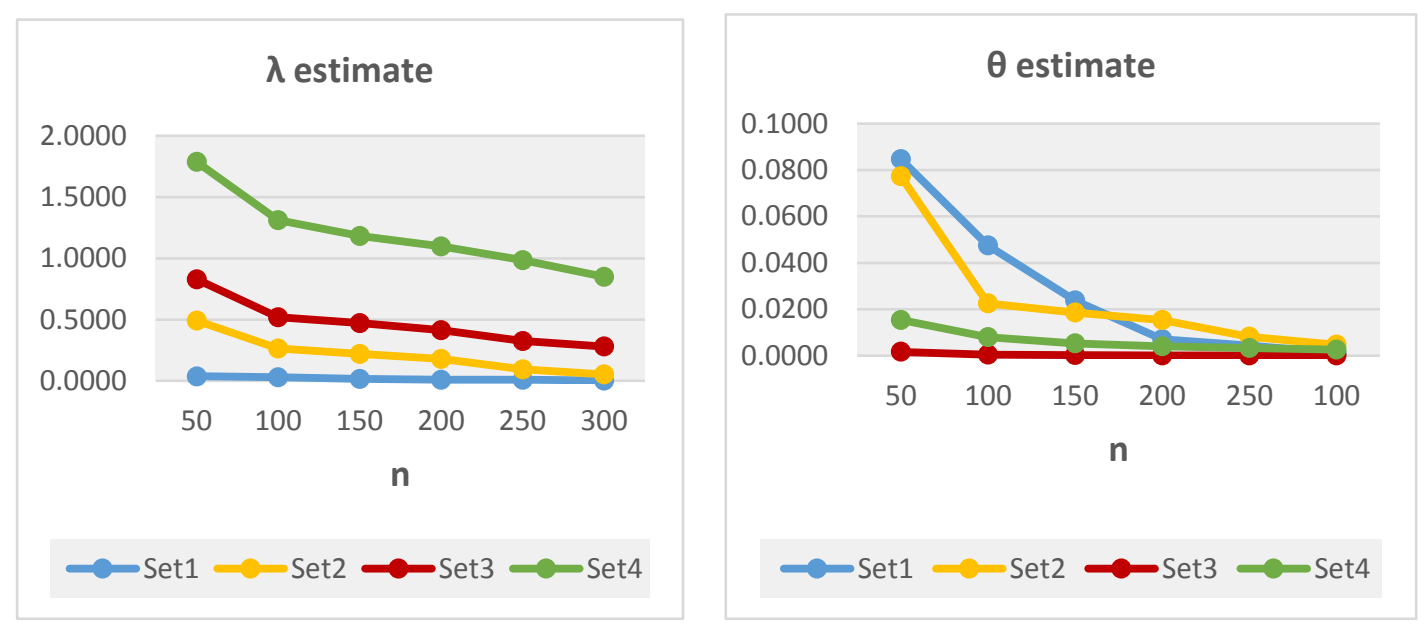

Figure 8. MSEs of $\lambda$ for all set of Figure 9. MSEs of $\theta$ for all set of parameters parameters 
Table 2. MSEs, RBs and SEs for Set1 and Set 2 of the IEL distribution via TIC

\begin{tabular}{|c|c|c|c|c|c|c|c|}
\hline \multirow[b]{2}{*}{$n$} & \multirow[b]{2}{*}{ Properties } & \multicolumn{3}{|c|}{$(\alpha=0.5, \lambda=0.8, \theta=2)$} & \multicolumn{3}{|c|}{$(\alpha=0.3, \lambda=2, \theta=1.5)$} \\
\hline & & $\hat{\alpha}$ & $\hat{\lambda}$ & $\hat{\theta}$ & $\hat{\alpha}$ & $\hat{\lambda}$ & $\hat{\theta}$ \\
\hline \multirow{3}{*}{50} & MSE & 0.0294 & 0.0383 & 0.0847 & 0.0091 & 0.4532 & 0.0773 \\
\hline & RB & 0.3410 & 0.2435 & 0.1392 & 0.3142 & 0.3354 & 0.1803 \\
\hline & SE & 0.0004 & 0.0004 & 0.0017 & 0.0130 & 0.0580 & 0.0650 \\
\hline \multirow{3}{*}{100} & MSE & 0.0225 & 0.0298 & 0.0474 & 0.0079 & 0.2339 & 0.0225 \\
\hline & RB & 0.2994 & 0.2135 & 0.1043 & 0.2963 & 0.2395 & 0.0949 \\
\hline & SE & 0.0001 & 0.0002 & 0.0006 & 0.0067 & 0.0660 & 0.0480 \\
\hline \multirow{3}{*}{150} & MSE & 0.0192 & 0.0172 & 0.0238 & 0.0073 & 0.2041 & 0.0186 \\
\hline & RB & 0.2770 & 0.1616 & 0.0734 & 0.2853 & 0.2239 & 0.0876 \\
\hline & SE & 0.0000 & 0.0001 & 0.0003 & 0.0005 & 0.0590 & 0.0370 \\
\hline \multirow{3}{*}{200} & MSE & 0.0183 & 0.0099 & 0.0070 & 0.0070 & 0.1704 & 0.0154 \\
\hline & RB & 0.2704 & 0.1217 & 0.0362 & 0.2781 & 0.2046 & 0.0803 \\
\hline & SE & 0.0000 & 0.0001 & 0.0002 & 0.0034 & 0.0540 & 0.0310 \\
\hline \multirow{3}{*}{250} & MSE & 0.0177 & 0.0080 & 0.0043 & 0.0069 & 0.0876 & 0.0082 \\
\hline & RB & 0.2659 & 0.1094 & 0.0272 & 0.2706 & 0.1459 & 0.0579 \\
\hline & SE & 0.0000 & 0.0001 & 0.0000 & 0.0027 & 0.0490 & 0.0260 \\
\hline \multirow{3}{*}{300} & MSE & 0.0173 & 0.0028 & 0.0012 & 0.0065 & 0.0498 & 0.0047 \\
\hline & $\mathrm{RB}$ & 0.2630 & 0.0624 & 0.0055 & 0.2680 & 0.1093 & 0.0431 \\
\hline & SE & 0.0000 & 0.0001 & 0.0001 & 0.0008 & 0.0010 & 0.0000 \\
\hline
\end{tabular}

Table 3. MSEs, RBs and SEs for Set3 and Set 4 of the IEL distribution via TIC

\begin{tabular}{|c|c|c|c|c|c|c|c|}
\hline \multirow[b]{2}{*}{$n$} & \multirow[b]{2}{*}{ Properties } & \multicolumn{3}{|c|}{$(\alpha=1.5, \lambda=0.5, \theta=0.4)$} & \multicolumn{3}{|c|}{$(\alpha=2, \lambda=1.5, \theta=0.8)$} \\
\hline & & $\hat{\alpha}$ & $\hat{\lambda}$ & $\hat{\theta}$ & $\hat{\alpha}$ & $\hat{\lambda}$ & $\hat{\theta}$ \\
\hline \multirow{3}{*}{50} & MSE & 0.1738 & 0.3377 & 0.0016 & 0.4805 & 0.9579 & 0154 \\
\hline & RB & 0.2742 & 1.1608 & 0.0890 & 0.3445 & 0.6512 & 0.1464 \\
\hline & SE & 0.0680 & 0.0280 & 0.0180 & 0.0760 & 0.0600 & 0.0410 \\
\hline \multirow{3}{*}{100} & MSE & 0.0227 & 0.2545 & 0.0004 & 0.1607 & 0.7940 & 0.0080 \\
\hline & $\mathrm{RB}$ & 0.0971 & 1.0077 & 0.0411 & 0.1993 & 0.5932 & 0.1086 \\
\hline & SE & 0.0390 & 0.02 & $\overline{0.0}$ & 0.0420 & 0.0460 & 0.0220 \\
\hline \multirow{3}{*}{150} & MSE & 0.0038 & 0.2 & 0.0002 & 0.0715 & 0.7082 & 0.0052 \\
\hline & RB & 0.0366 & 1.0022 & 0.0320 & 0.1328 & 0.5604 & 0.0878 \\
\hline & SE & 0.0290 & 0.0240 & 0.0065 & 0.0310 & 0.0400 & 0.0150 \\
\hline \multirow{3}{*}{200} & MSE & 0.0017 & 0.2343 & 0.0001 & 0.0603 & 0.6830 & 0.0041 \\
\hline & $\mathrm{RB}$ & 0.0227 & 0.9672 & 0.0244 & 0.1222 & 0.5506 & 0.0788 \\
\hline & SE & 0.0220 & 0.0200 & 0.0049 & 0.0230 & 0.0310 & 0.0110 \\
\hline \multirow{3}{*}{250} & MSE & 0.0004 & 0.2312 & 0.0001 & 0.0486 & 0.6575 & 0.0033 \\
\hline & $\mathrm{RB}$ & 0.0520 & 0.9611 & 0.0213 & 0.1098 & 0.5403 & 0.0714 \\
\hline & SE & 0.0190 & 0.0180 & 0.0040 & 0.0190 & 0.0270 & 0.0090 \\
\hline \multirow{3}{*}{300} & MSE & 0.0003 & 0.2272 & 0.0001 & 0.0467 & 0.5695 & 0.0026 \\
\hline & $\mathrm{RB}$ & 0.0174 & 0.9529 & 0.0174 & 0.1077 & 0.5029 & 0.0630 \\
\hline & SE & 0.0160 & 0.0150 & 0.0033 & 0.0170 & 0.0240 & 0.0075 \\
\hline
\end{tabular}


Table 4. LCB, UCB and Length of the estimates for Set 1 and Set 2 of the IEL distribution

\begin{tabular}{|c|c|c|c|c|c|c|c|}
\hline \multirow[b]{2}{*}{$n$} & \multirow{2}{*}{ Properties } & \multicolumn{3}{|c|}{$(\alpha=0.5, \lambda=0.8, \theta=2)$} & \multicolumn{3}{|c|}{$(\alpha=0.3, \lambda=2, \theta=1.5)$} \\
\hline & & LCB & UCB & Length & LCB & UCB & Length \\
\hline \multirow{3}{*}{50} & $\alpha$ & 0.6650 & 0.6760 & 0.0110 & 0.3680 & 0.4200 & 0.0520 \\
\hline & $\lambda$ & 0.6000 & 0.6110 & 0.0110 & 0.9426 & 1.2160 & 0.2734 \\
\hline & $\theta$ & 1.6980 & 1.7450 & 0.0470 & 1.1030 & 1.3560 & 0.2530 \\
\hline \multirow{3}{*}{100} & $\alpha$ & 0.6480 & 0.6520 & 0.0040 & 0.3760 & 0.4020 & 0.0260 \\
\hline & $\lambda$ & 0.6240 & 0.6340 & 0.0097 & 1.3920 & 1.6500 & 0.2590 \\
\hline & $\theta$ & 1.7790 & 1.8040 & 0.0250 & 1.2640 & 1.4510 & 0.1870 \\
\hline \multirow{3}{*}{150} & $\alpha$ & 0.6370 & 0.6400 & 0.0022 & 0.3770 & 0.3940 & 0.0180 \\
\hline & $\lambda$ & 0.6670 & 0.6740 & 0.0067 & 1.4360 & 1.6680 & 0.2320 \\
\hline & $\theta$ & 1.8460 & 1.8610 & 0.0150 & 1.2970 & 1.4410 & 0.1440 \\
\hline \multirow{3}{*}{200} & $\alpha$ & 0.6340 & 0.6360 & 0.0014 & 0.3770 & 0.3900 & 0.0130 \\
\hline & $\lambda$ & 0.7000 & 0.7050 & 0.0055 & 1.4850 & 1.6970 & 0.2120 \\
\hline & $\theta$ & 1.9220 & 1.9330 & 0.0110 & 1.3200 & 1.4390 & 0.1200 \\
\hline \multirow{3}{*}{250} & $\alpha$ & 0.6320 & 0.6330 & 0.0010 & 0.3760 & 0.3860 & 0.0110 \\
\hline & $\lambda$ & 0.7100 & 0.7150 & 0.0047 & 1.6120 & 1.8050 & 0.1930 \\
\hline & $\theta$ & 1.9410 & 1.9500 & 0.0090 & 1.3620 & \begin{tabular}{|l|}
1.4650 \\
\end{tabular} & 0.1030 \\
\hline \multirow{3}{*}{300} & $\alpha$ & 0.6310 & 0.6320 & 0.0008 & 0.3760 & 0.3850 & 0.0088 \\
\hline & $\lambda$ & 0.7480 & 0.7520 & 0.0039 & 1.6930 & 1.8690 & 0.1760 \\
\hline & $\theta$ & 1.9850 & 1.9930 & 0.0073 & 1.3900 & 1.4810 & 0.0910 \\
\hline
\end{tabular}

Table 5. LCB, UCB and Length of the estimates for Set 3 and Set 4 of the IEL distribution

\begin{tabular}{|c|c|c|c|c|c|c|c|}
\hline \multirow{2}{*}{$n$} & \multirow{2}{*}{ Properties } & \multicolumn{3}{|c|}{$(\alpha=1.5, \lambda=0.5, \theta=0.4)$} & \multicolumn{3}{|c|}{$(\alpha=2, \lambda=1.5, \theta=0.8)$} \\
\hline & & LCB & UCB & Length & LCB & UCB & Length \\
\hline \multirow{3}{*}{50} & $\alpha$ & 1.7780 & 2.0450 & 0.2670 & 2.5400 & 2.8380 & 0.2990 \\
\hline & $\lambda$ & 1.0250 & 1.1360 & 0.1110 & 2.3590 & 2.5950 & 0.2360 \\
\hline & $\theta$ & 0.4000 & 0.4710 & 0.0710 & 0.8380 & 0.9970 & 0.1590 \\
\hline \multirow{3}{*}{100} & $\alpha$ & 0.3980 & 0.4350 & 0.1520 & 2.3160 & 2.4810 & 0.1640 \\
\hline & $\lambda$ & 0.9540 & 1.0540 & 0.1000 & 2.3000 & 2.4800 & 0.1800 \\
\hline & $\theta$ & 0.3980 & 0.4350 & 0.0370 & 0.8450 & 0.9290 & 0.0850 \\
\hline \multirow{3}{*}{150} & $\alpha$ & 1.4990 & 1.6110 & 0.1120 & 2.2050 & 2.3260 & 0.1200 \\
\hline & $\lambda$ & 0.9530 & 1.0490 & 0.0960 & 2.2620 & 2.4190 & 0.1570 \\
\hline & $\theta$ & 0.4000 & 0.4260 & 0.0250 & 0.8410 & 0.8990 & 0.0580 \\
\hline \multirow{3}{*}{200} & $\alpha$ & 1.4900 & 1.5780 & 0.0880 & 2.1980 & 2.2900 & 0.0920 \\
\hline & $\lambda$ & 0.9430 & 1.0240 & 0.0800 & 2.2650 & 2.3870 & 0.1230 \\
\hline & $\theta$ & 0.4000 & 0.4190 & 0.0190 & 0.8410 & 0.8850 & 0.0440 \\
\hline \multirow{3}{*}{250} & $\alpha$ & 1.4710 & 1.5440 & \begin{tabular}{|l|}
0.0730 \\
\end{tabular} & 2.1820 & 2.2570 & 0.0760 \\
\hline & $\lambda$ & 0.9460 & 1.0150 & 0.0700 & 2.2580 & 2.3630 & 0.1050 \\
\hline & $\theta$ & 0.4010 & 0.4160 & 0.0160 & 0.8400 & 0.8750 & 0.0350 \\
\hline \multirow{3}{*}{300} & $\alpha$ & 1.4750 & 1.5360 & 0.0610 & 2.1820 & 2.2490 & 0.0670 \\
\hline & $\lambda$ & 0.9470 & 1.0060 & 0.0590 & 2.2070 & 2.3010 & 0.0940 \\
\hline & $\theta$ & 0.4010 & 0.4130 & 0.0130 & 0.8650 & 0.8650 & 0.0290 \\
\hline
\end{tabular}




\section{REAL DATA APPLICATIONS}

We fit the IEL distribution to two different real data sets and we check the behavior with those of the inverse L (IL), TEL, inverted exponentiated Rayleigh (IER), inverse Weibull (IW) and Kumarswamy exponentiated L (KEL). In each real data set, the ML estimates and their corresponding SEs of the model parameters are obtained. The model selection is conducted using; -2 log-likelihood (-2logl), Akaike information criterion (AIC), the consistent AIC (CAIC), Bayesian information criterion (BIC), HannanQuinn information criterion (HQIC), Cramer-von Mises (W*) statistic, Kolmogorov-Smirnov (K-S) statistic, and Anderson-Darling $\left(\mathrm{A}^{*}\right)$ statistic. However, the better distribution corresponds to the smaller values of the previous measures. Furthermore, the histogram and the estimated pdf for the models are displayed for each data set. Moreover, the empirical cdf and estimated pdf for the models are displayed for both real data.

Data Set 1: The first data set represents the number of million revolutions of the 23 ball bearings before failure ([25]). Table 6 gives the ML estimates of the model parameters and their SEs (in the parentheses) for 23 ball bearings before failure. The results in Table 7 indicate that the IEL model is suitable for this data set based on the selected criteria. The IEL model has the smallest goodness of fit measures.

Table 6. ML estimates and SEs for the first data

\begin{tabular}{|l|l|l|l|l|l|l|l|}
\hline Distribution & $\hat{\lambda}$ & $\hat{\theta}$ & $\hat{\alpha}$ & $\hat{\gamma}$ & $\hat{\beta}$ & $\hat{a}$ & $\hat{b}$ \\
\hline IEL & 51.071 & 12.299 & 5.018 & - & - & - & - \\
& $(10.069)$ & $(1.558)$ & $(1.696)$ & - & - & - & - \\
\hline IL & 119.394 & - & 0.461 & - & - & - & - \\
& $(299.793)$ & - & $(0.892)$ & - & - & - & - \\
\hline TEL & 0.491 & 2.671 & 21.538 & 0.036 & - & - & - \\
& $(0.547)$ & $(1.163)$ & $(27.976)$ & $(0.046)$ & - & - & - \\
\hline IER & - & - & 0.605917 & - & 946.054337 & - & - \\
& - & - & $(0.146)$ & - & $(325.895)$ & - & - \\
\hline IW & 48.575 & - & 1.834 & - & - & - & - \\
& $(5.866)$ & - & $(0.269)$ & - & - & - & - \\
\hline KEL & 2.325 & 0.565 & 48.382 & - & - & 1.282 & 31.478 \\
& $(7.064)$ & $(0.44)$ & $(65.078)$ & - & - & $(1.724)$ & $(75.744)$ \\
\hline
\end{tabular}

Table 7. Goodness of fit measures for the first data

\begin{tabular}{|l|l|l|l|l|l|l|l|l|l|}
\hline Distribution & -2 LogL & AIC & BIC & CAIC & HQIC & $W^{*}$ & $A^{*}$ & K-S & $\begin{array}{l}\text { P- } \\
\text { value }\end{array}$ \\
\hline IEL & 226.046 & 232.046 & 235.452 & 233.309 & 232.902 & 0.030 & 0.190 & 0.088 & 0.994 \\
\hline IL & 243.577 & 247.577 & 249.848 & 248.177 & 248.149 & 0.274 & 3.927 & 0.305 & 0.027 \\
\hline TEL & 228.764 & 236.764 & 241.306 & 238.986 & 237.906 & 0.048 & 0.354 & 0.108 & 0.950 \\
\hline IER & 238.411 & 242.411 & 244.682 & 243.011 & 242.982 & 0.117 & 1.297 & 0.276 & 0.060 \\
\hline IW & 231.561 & 235.561 & 237.832 & 236.161 & 236.132 & 0.066 & 0.520 & 0.190 & 0.810 \\
\hline KEL & 226.120 & 236.120 & 241.797 & 239.649 & 237.547 & 0.031 & 0.192 & 1.000 & 0.000 \\
\hline
\end{tabular}

It is also clear from Figure 10 that the IEL distribution provides a better fit and therefore be one of the best models for this data set. 

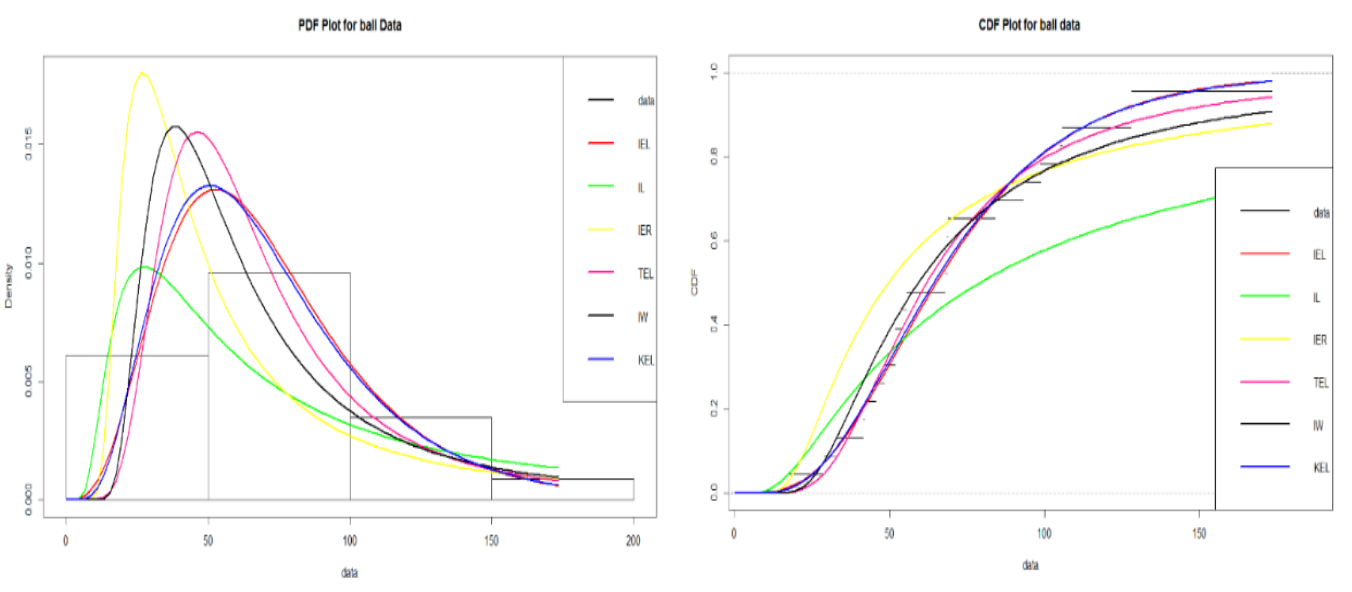

Figure 10. Estimated pdfs and cdfs of models for 23 ball bearings before failure

Data Set 2: The second data set represents remission times (in months) of a random sample of 128 bladder cancer patient's [26]. Table 8 gives the ML estimates of the model parameters and their SEs (in the parentheses) for the 128 bladder cancer patient's. The results in Table 9 indicate that the IEL model is suitable for this data set based on the selected criteria. The IEL model has the smallest values corresponding to other models.

Table 8. ML estimates and SEs for the second data

\begin{tabular}{|l|l|l|l|l|l|l|l|}
\hline Distribution & $\hat{\lambda}$ & $\hat{\theta}$ & $\hat{\alpha}$ & $\hat{\gamma}$ & $\hat{\beta}$ & $\hat{a}$ & $\hat{b}$ \\
\hline IEL & 30.214 & 5.079 & 1.083 & - & - & - & - \\
& $(3.464)$ & $(0.046)$ & $(0.106)$ & - & - & - & - \\
\hline IL & 1.531 & - & 2.782 & - & - & - & - \\
& $(0.304)$ & - & $(0.818)$ & - & - & - & - \\
\hline TEL & 0.257 & 6.482 & 1.107 & 0.02 & - & - & - \\
& $(0.315)$ & $(1.098)$ & $(0.231)$ & $(0.001774)$ & - & - & - \\
\hline IER & - & - & 0.183 & - & 0.094 & - & - \\
& - & - & $(0.018)$ & - & $(0.02)$ & - & - \\
\hline IW & 2.287 & - & 0.69 & - & - & - & - \\
& $(0.312)$ & - & $(0.042)$ & - & - & - & - \\
\hline \multirow{2}{*}{ KEL } & 3.172 & 0.087 & 1.43 & - & - & 2.138 & 78.45 \\
& $(5.013)$ & $(0.038)$ & $(0.297)$ & - & - & $(0.444)$ & $(29.662)$ \\
\hline
\end{tabular}

Table 9. Goodness of fit measures for the second data

\begin{tabular}{|l|l|l|l|l|l|c|c|l|l|}
\hline Distribution & $-2 \operatorname{LogL}$ & AIC & BIC & CAIC & HQIC & $W^{*}$ & $A^{*}$ & K-S & P-value \\
\hline IEL & 801.096 & 807.096 & 815.652 & 807.290 & 810.573 & 0.051 & 0.341 & 0.046 & 0.948 \\
\hline IL & 824.528 & 828.528 & 834.232 & 828.624 & 830.845 & 0.414 & 3.131 & 0.103 & 0.133 \\
\hline TEL & 842.018 & 850.018 & 861.426 & 850.343 & 854.653 & 0.058 & 0.377 & 0.256 & 0.000 \\
\hline IER & 938.469 & 942.469 & 948.174 & 942.565 & 944.787 & 1.634 & 8.940 & 0.313 & 0.000 \\
\hline IW & 857.352 & 861.352 & 867.056 & 861.448 & 863.669 & 0.944 & 5.508 & 0.995 & 0.005 \\
\hline KEL & 816.565 & 824.565 & 835.973 & 824.891 & 829.201 & 0.382 & 2.163 & 1.000 & 0.000 \\
\hline
\end{tabular}



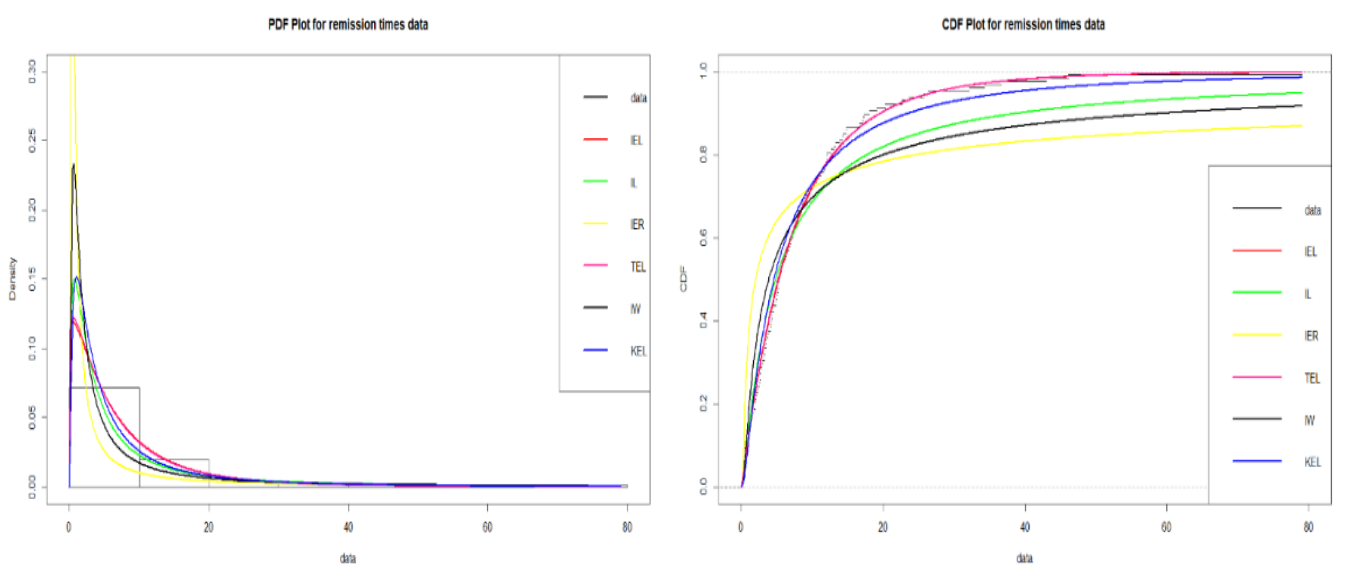

Figure 11. Estimated $p d f s$ and cdfs of models for 128 bladder cancer patient's

It is also clear from Figure 11 that the IEL distribution provides a better fit and therefore be one of the best models for this data set.

\section{CONCLUDING REMARKS}

In this paper, three-parameter model, called the inverted exponentiated Lomax distribution is proposed and discussed. Some of statistical properties of the subject model for instance, quantile measures, moments, Rényi entropy and distribution of OS are obtained. The ML method is implemented for estimating population parameter depending on TIC sample. Also, the approximate CIs are obtained. The simulation study is implemented to check the performance of the estimators. Practical relevance and applicability of the IEL distribution are illustrated via real data sets. The real life application indicates that the IEL model produces a good fit than the other competitive models.

\section{ACKNOWLEDGMENTS}

The authors are grateful to the editor and referees for their useful comments and suggestions for improvement which undoubtedly enhanced the presentation and quality of the article.

\section{CONFLICTS OF INTEREST}

No conflict of interest was declared by the authors.

\section{REFERENCES}

[1] Dubey, S., “Compound gamma, beta and F distributions”. Metrika, 16(1):27-31, (1970).

[2] Bryson, M.C., "Heavy-tailed distributions: properties and tests". Technimetrics, 16: 61-68, (1974).

[3] Myhre, J., Saunders, S., "Screen testing and conditional probability of survival, In: Crowley, J. and Johnson, R.A., eds. Survival Analysis". Lecture Notes-Monograph Series. Institute of Mathematical Statistics, 2: 166-178, (1982).

[4] Ahsanullah, M., "Record values of the Lomax distribution". Statistica Neerlandica, 45: 21-29, (1991).

[5] Balakrishnan, N., Ahsanullah, M., "Relations for single and product moments of record values from Lomax distribution". Sankhya B, 56: 140-146, (1994). 
[6] Abd-Elfattah, A.M., Alaboud, F.M., Alharbey, H.A., "On sample size estimation for Lomax distribution". Australian Journal of Basic and Applied Sciences, 1(4): 373-378, (2007).

[7] Hassan A.S., Al-Ghamdi, A.S., "Optimum step stress accelerated life testing for Lomax distribution". Journal of Applied Sciences Research, 5(12):1-12, (2009).

[8] Ashour, S.K., Abdelfattah, A.M., Mohammad, B.S.K., "Parameter estimation of the hybrid censored Lomax distribution". Pakistan Journal of Statistics and Operations Research, 7: 1-19, (2011).

[9] Hassan, A.S., Assar, S.M., Shelbaia, A., "Optimum step stress accelerated life test plan for Lomax distribution with an adaptive type-II progressive hybrid censoring". British Journal of Mathematics and Computer Science, 13(2): 1-19, (2016).

[10] Ghitany, M.E., Al-Awadhi, F.A., Alkhalfan, L.A., "Marshall-Olkin extended Lomax distribution and its application to censored data". Communication in Statistics-Theory and Methods, 36(10): 18551866, (2007).

[11] Gupta, R.C., Ghitany, M.E., Al-Mutairi, D.K., "Estimation of reliability from Marshall-Olkin extended Lomax distributions". Journal of Statistical Computation and Simulation, 80(8): 937-947, (2010).

[12] Abdul-Moniem, I.B., Abdel-Hameed, H.F., "On exponentiated Lomax distribution". International Journal of Mathematical Education, 33(5): 1-7, (2012).

[13] Ashour, S.K., Eltehiwy, M.A., "Transmuted exponentiated Lomax distribution". Australian Journal of Basic and Applied Sciences, 7: 658-667, (2013).

[14] Al-Zahrani, B., "An extended Poisson-Lomax distribution". Advances in Mathematics: Scientific Journal, 4(2): 79-89, (2015).

[15] El-Bassiouny, A., Abdo, N., Shahen, H., "Exponential Lomax distribution". International Journal of Computer Applications, 121(13):975-8887, (2015).

[16] Tahir, M.H., Cordeiro, G.M., Mansoor, M., Zubair, M., "The Weibull-Lomax distribution: properties and applications". Hacettepe Journal of Mathematics and Statistics, 44 (2): 461-480, (2015).

[17] Rady, E.A., Hassanein, W.A., Elhaddad, T A., "The power Lomax distribution with an application to bladder cancer data". Springer Plus, 5:1838DOI 10.1186/s40064-016-3464-y, (2016).

[18] Hassan, A.S., Abd-Alla, M., "Exponentiated Lomax geometric distribution: properties and applications". Pakistan Journal of Statistics and Operation Research, 13(3): 545-566, (2017).

[19] Hassan, A.S., Nassr, S.G., "Power Lomax Poisson distribution: properties and estimation". Journal of Data Science, 18(1):105-128, (2018).

[20] Hassan, A.S., Abd-Allah, M., "Exponentiated Weibull-Lomax distribution: properties and estimation". Journal of Data Science, 16 (2): 277-298, (2018).

[21] Hassan, A.S., Abd-Allah, M., "On the inverse power Lomax distribution". Annals of Data Science, 6(2): 259-278, (2019).

[22] Kenney, J.F., Keeping, E., "Mathematics of Statistics”. D. Van Nostrand Company, (1962).

[23] Moors, J.J.A., "A quantile alternative for kurtosis". Journal of the Royal Statistical Society. Series D (The Statistician), 37(1):25-32, (1988). 
[24] David, H.A., "Order statistics", Second edition, Wiley, New York, (1981).

[25] Lawless, J.F., "Statistical Models and Methods Lifetime Data, Wiley, New York, (1982).

[26] Lee, E.T., Wang, J.W., "Statistical Methods for Survival Data Analysis". $3^{\text {rd }}$ edition, Wiley, New York, http://dx.doi.org/10.1002/0471458546, (2003). 